\title{
Discrete Element Modeling of Hemp Particle Separation Using a 3D Vibratory Separator
}

\author{
M.A. Sadek ${ }^{1}$ and Y. Chen ${ }^{1, *}$ \\ ${ }^{I}$ Department of Biosystems Engineering, University of Manitoba, Winnipeg, MB, R3T 5 V6 Canada \\ *Email: ying.chen@umanitoba.ca \\ http://dx.doi.org/10.7451/CBE.2013.55.2.23
}

Sadek, M.A., and Y. Chen. 2013. Discrete Element Modeling of Hemp Particle Separation Using a 3D Vibratory Separator. Canadian Biosystems Engineering/Le génie des biosystèmes au Canada 55: 2.23-2.31. Existing cleaning equipment was ineffective for cleaning processed hemp material (a mixture of fibre, core, and chaff). One of the major obstacles was the lack of the knowledge on the cleaning process. In this study, a 3D vibratory separator (screen-type) was experimentally studied for cleaning processed hemp material through separating chaffs (small particles) from the other particles (larger particles) in a mixture. In the experiment, the separation efficiency of the $3 \mathrm{D}$ vibratory separator was measured for different horizontal and vertical vibratory settings of the separator. Results showed that the separation efficiency varied from 23 to $86 \%$, depending on the vibratory settings. The low separation efficiency was due to the fact that chaff was held within the entangled fibre, which prevented chaff from passing through the screen. A numerical model was developed to simulate the $3 \mathrm{D}$ vibratory separator using the discrete element method (DEM). The main model components included virtual particles of processed hemp material as well as a virtual $3 \mathrm{D}$ vibratory separator. The model is able to deal with mixtures of different particle sizes and different portions of fibre, core, and chaff particles, and predict the separation rates and efficiencies of the 3D vibratory separator. When compared with the measured separation rate, the simulated separation rate was higher towards the end of separation processes due to inexistence of fibre tangle problem in the virtual hemp particles. However, the trend of simulated values could adequately explain that of the measurements. Keywords: Hemp, separation, vibratory shaker, discrete element method, simulation.

Les équipements de nettoyage qui sont sur le marché sont inefficaces pour le nettoyage du chanvre récolté (un mélange de fibres, tiges et balle). Un des obstacles majeurs vient du manque de connaissance du processus de nettoyage. Dans cette étude, un crible vibrant 3D (tamis) a été testé pour nettoyer du chanvre récolté en séparant la balle (petites particules) des autres particules (grosses particules) du mélange. Dans l'expérience, l'efficacité de séparation du crible a été mesurée pour différents ajustements verticaux et horizontaux. Les résultats ont montré que l'efficacité de séparation variait entre 26 et $86 \%$ selon le niveau de vibration. L'efficacité de séparation la plus faible était obtenue lorsque la balle était enchevêtrée à la fibre ce qui empêchait la balle de passer à travers le tamis. La méthode des éléments discrets (DEM) a été utilisée pour développer un modèle numérique qui simule le crible vibrant 3D. Les principaux éléments du modèle incluent des particules virtuelles de chanvre récolté et un crible vibrant $3 \mathrm{D}$ virtuel. Le modèle peut tenir compte de mélanges de différentes tailles de particules et de différentes proportions de fibre, tiges et balle, et prédire le taux de séparation et l'efficacité du crible vibrant 3D. Lorsque comparé aux taux de séparation mesurés, ceux simulés étaient plus élevés vers la fin du processus de séparation car il n'y avait pas de problème d'enchevêtrement des fibres dans le mélange de chanvre virtuel. Toutefois, la tendance des valeurs simulées représentait adéquatement les valeurs mesurées. Mots clés: chanvre, séparation, crible vibrant, méthode des éléments discrets, simulation.

\section{INTRODUCTION}

Current demands for hemp fibre are rapidly increasing due to the diverse use of natural fibres. Obtaining hemp fibre requires two main processes: decortication (mechanically processing hemp for fibre) and post-decortication fibre cleaning. Decorticated hemp from the decortication process consists of different fractions: fibre (the outer layer of hemp stem), core, also named as hurds (the inner layer of hemp stem), and chaff (small fibre and core particles). The post-decortication fibre cleaning is to remove core and chaff from the mixture to obtain clean fibre, which was the goal of this study. This goal is typically achieved in several stages. The first stage is to separate the chaff and other particles, and the following stage is to further separate the fibre and core particles. The reason for using different stages is that different separating principles should be used for effective separations. This study focused on the first stage, i.e. separate chaff from processed hemp, which can be done using the common screening method (Fürll and Hempel 2000). The second stage for separating hemp fibre and core particles has been studied by Parvin et al. (2013) using carding, flotation, and aerodynamic methods. Cleaning equipment (such as cylinder cleaners, impact cleaners, trashmaster) used in cotton industry, adopted for flax fibre (Anthony 2002; 2005), may also be used for the second stage cleaning of hemp.

The screening method has been widely used for separation of particles with different sizes (Cleary et al. 2009; Vorster et al. 2002). One of the common screening machines is vibratory separator (Parvin et al. 2013), and it has been adopted in separation of hemp materials. Münder et al. (2004) use a vibratory separator to eliminate chaff from long fibres. Pecenka and Furll (2008) have reported a vibratory separator with oscillating comb for fibre cleaning. The combing action helped loose fibres for easy removing of chaff. To date, little research has been done to investigate the efficiency of the screening method for separations of processed hemp.

In this study, separation of decorticated hemp material with a 3D vibratory separator was investigated using both experimental and modeling approaches. The discrete element method (DEM) was used for the modeling. The DEM is a numerical approach to simulate material as discrete assemblages of particles. Cundall and Strack (1979) was first introduced the DEM for analyzing geological materials (rocks and soils) through their constituent of individual particles. Since then, the DEM has been used to model many other materials. A relevant study 
was simulations of separation of paddy and brown rice grains by Sakaguchi et al. (2001). In their study, a 2D model was developed using the DEM to predict the segregation behaviours of these two different rice grains, which were comparable with measurements. Their model used spherical particles to represent rice grains, and the working principle of the model was using the differences in particle density for particle separation. Whereas in this study, clusters of spherical particles were used to represent decorticated hemp particles, and the working principle of the model was using the differences in particle size for particle separation.

This study used a common commercial DEM software, the Particle Flow Code in Three Dimension $\left(\mathrm{PFC}^{3 \mathrm{D}}\right)$, developed by Itasca Consulting Group Inc., Minneapolis, MN. In $\mathrm{PFC}^{3 \mathrm{D}}$, material to be simulated is represented by assemblies of individual particles. The basic particles are "balls". $\mathrm{PFC}^{3 \mathrm{D}}$ allows users to model material particles with any arbitrary shapes by attaching a group of balls together using the PFC ${ }^{3 \mathrm{D}}$ bond models. In $\mathrm{PFC}^{3 \mathrm{D}}$, "walls" simulate the "surrounding environment" in which the material is placed, such as boundaries and machine surfaces. In simulating a ferrohydrostatic separator, Murariu and Sergeant (2007) have demonstrated that $\mathrm{PFC}^{3 \mathrm{D}}$ is an effective modeling tool for understanding the flow behaviour of gravel particles (balls) inside the separation chamber (consisting of walls) under different feeding rates and particle sizes. These features of $\mathrm{PFC}^{3 \mathrm{D}}$ allow for effective simulations of the irregular shapes of hemp particles and their interactions with the $3 \mathrm{D}$ vibratory separator.

The objective of this study was to investigate the postdecortication process for separation of hemp materials using a $3 \mathrm{D}$ vibratory separator. The specific objectives were to 1) measure the separation efficiency under different operating conditions, 2) develop a model to simulate the separation process using $\mathrm{PFC}^{3 \mathrm{D}}$, and 3 ) to validate the model and use the model to predict the separation rate and efficiency.

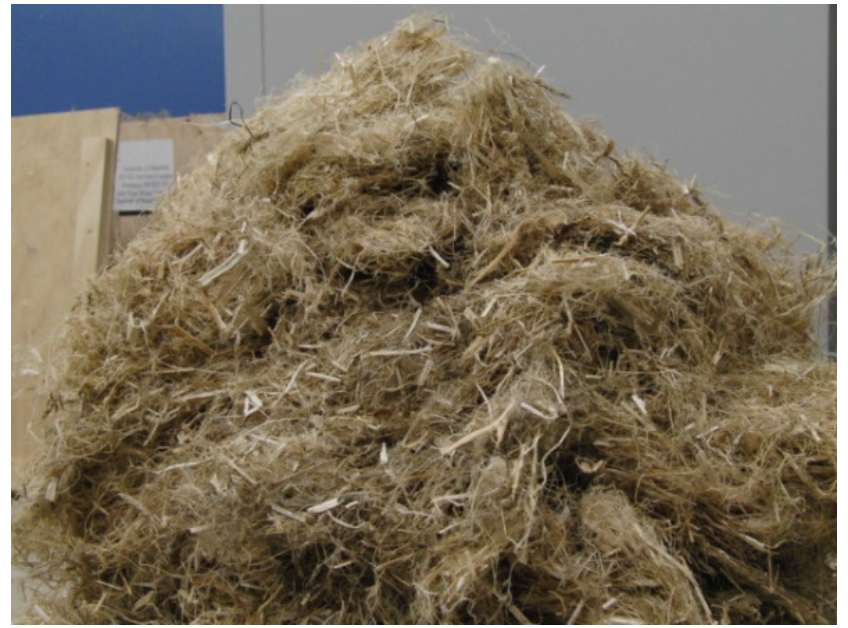

Fig. 1. Decorticated hemp material used for the separation experiment.

\section{METHODOLOGY}

\section{Hemp separation experiment}

Feedstock used for the separation experiment The feedstock used for the separation experiment was decorticated hemp material collected from a hemp processing plant in Manitoba, Canada. The feedstock contained fibre, cores, and chaff as shown in Fig. 1. Detail compositions of the feedstock are described later in this paper.

The 3D vibratory separator The 3D vibratory separator used for separation experiment was SWECO shaker (Vibro Energy Separator - XS40, SWECO Canada, Inc., ON, Canada) (Fig. 2). The separator had several screen decks and a vibration motion generator. In the experiment, only one deck (20 mm screen opening size) was used because the purpose of the experiment was to remove the chaff only from the feedstock, and the chaff was defined as those particles smaller than $20 \mathrm{~mm}$ in this study. During separation tests, chaff would fall through the screen into the collection pan. The motion generator consisted of a motor with eccentric weights, which were adjustable, and springs, which supported the decks. Each setting of the centric weights corresponded to a horizontal and a vertical centrifugal force specified by the manufacturer of the separator. Those forces caused the screen deck to vibrate in a $3 \mathrm{D}$ motion.

Experimental design The separation efficiency of the separator was tested for nine treatments, which were the combinations of three different horizontal and vertical vibration settings of the separator. Under each vibration direction, the three levels of centrifugal force selected were the highest, medium (the recommended level by the manufacturer), and lowest. Each treatment was replicated four times. A total of 36 tests ( 3 horizontal settings $\times 3$

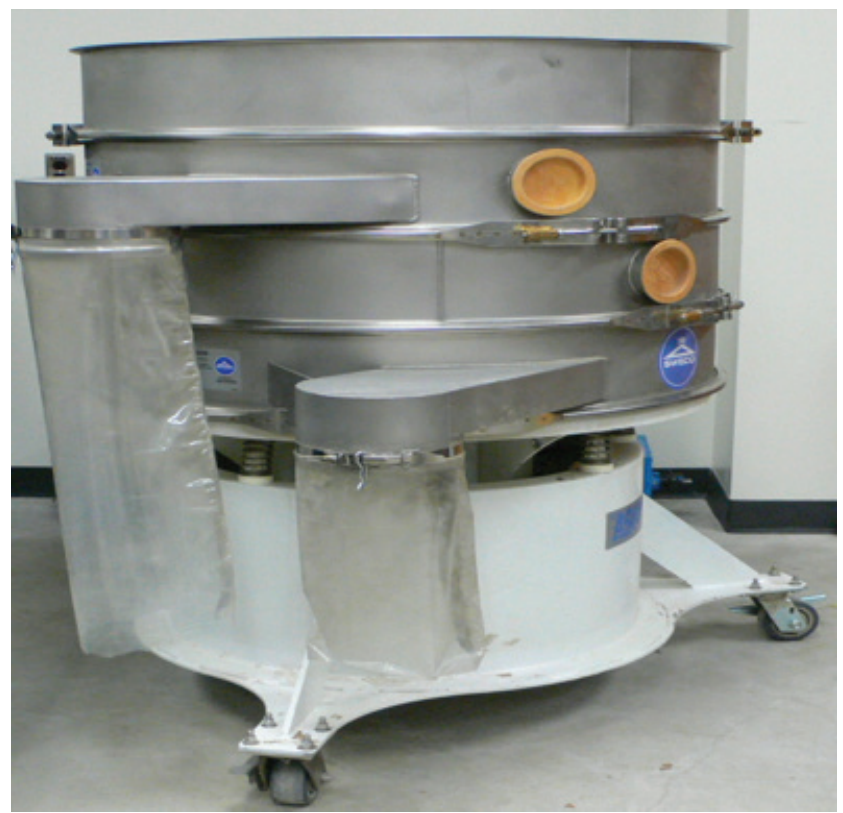

Fig. 2. The 3D vibratory separator used in the separation experiment. 
Table 1. Summary of the treatments.

\begin{tabular}{ccc}
\hline $\begin{array}{c}\text { Vibration } \\
\text { direction }\end{array}$ & $\begin{array}{c}\text { Force } \\
\text { setting }\end{array}$ & $\begin{array}{c}\text { Centrifugal force } \\
(\mathrm{N})^{*}\end{array}$ \\
\hline \multirow{3}{*}{ Horizontal } & H1 & 13367 \\
& H2 & 9920 \\
& H3 & 2678 \\
Vertical & V1 & 1174 \\
& V2 & 4346 \\
& V3 & 5858 \\
\hline
\end{tabular}

Specified by Sweco (2003).

vertical settings $\mathrm{x} 4$ replications) were performed in a completely random order. The corresponding centrifugal forces (Sweco 2003) for these treatments are listed in Table 1.

Test procedure and measurement Samples, each weighing $100 \mathrm{~g}$, were collected randomly from the feedstock shown in Fig. 1. Four samples were used for analysing the feedstock composition, i.e. the percentage of chaff, fibre, or core in the feedstock. This was important background information. The percentage of chaff in the feedstock is the base for determining the separation efficiency. The percentages of fibre and core affect separation efficiency. Fibre and core particles would behave differently in the separator. For example, fibre particles have tendency to tangle together, hold chaff, and prevent chaff from falling through screens.

For the composition analysis, each of the four samples was separated manually into the following three different fractions (Fig. 3), and the weight of the fraction was recorded to determine the percentage of each fraction in the mixture.

a) Fibre, fibres longer than $20 \mathrm{~mm}$;

b) Core, cores longer than $20 \mathrm{~mm}$;

c) Chaff, fibres and cores equal to or shorter than $20 \mathrm{~mm}$.

Before each test run, the vibration of the separator was set to the desire horizontal and vertical forces. A $100-\mathrm{g}$ sample was manually fed on the deck through the central opening of a lid on top of the screen deck. Then, the vibration commenced and continued for three minutes. At the end of the run, the portion of the material passed the screen was collected and weighed to determine the separation efficiency described in the section below. This measurement was performed for all 36 tests. In one test, the composition of the portion of the material passed the screen was analysed to see if any non-chaff material passed the screen. For model validation purpose, additional three tests were conducted to examine separation rates, in which the material passed the screen was collected multiple times during the period of three minutes at 30 -second intervals. During the test, the horizontal and vertical vibration amplitudes of the separator were read from the gauge stickers on the machine for the modeling purpose.

In particulate solids analyses, the mass of material, which is capable of passing a screen, is often determined based on the linear dimension of particle and the screen size. When the particle size is smaller than the screen opening size, the particle is considered to be capable of passing (referred as to passing particles). The separation efficiency is defined as the mass of all passing particles divided by that mass of the material which is capable of passing (Richardson et al. 2002). In this case, among the different fractions of the feedstock, the chaff was the only fraction, which was capable of passing the $20 \mathrm{~mm}$ screen. Therefore, the separation efficiency was determined as

$$
\eta=\frac{m_{p}}{m_{c}} \times 100
$$

where:

$\eta=$ separation efficiency (\%);

$m_{p}=$ mass of all passing particles $(\mathrm{g})$;

$m_{c h}=$ mass of the chaffs present in the feed $(\mathrm{g})$.

Data analysis Data of separation efficiency were analyzed using SAS 9.1.3. The experimental parameters were arranged into two factor factorial design. Scheffe's test was used to determine the differences among least square means. A significance level of 0.05 was applied to all the analysis.

\section{Model of the 3D vibratory separator}

Virtual hemp fibre and core Virtual hemp fibre and core were defined using the $\mathrm{PFC}^{3 \mathrm{D}}$ cluster logics, and the shapes of virtual fibre and core were determined based on the general features of the real fibre and core. The virtual fibre was a string shaped cluster consisting of balls

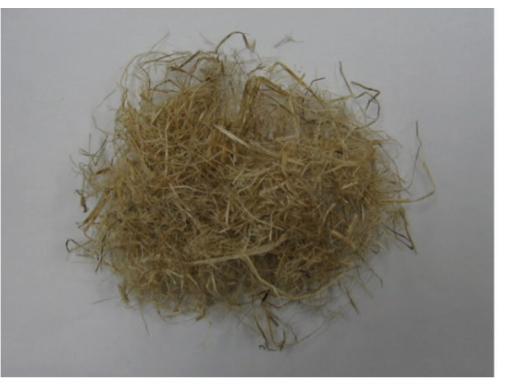

(a) Fibre

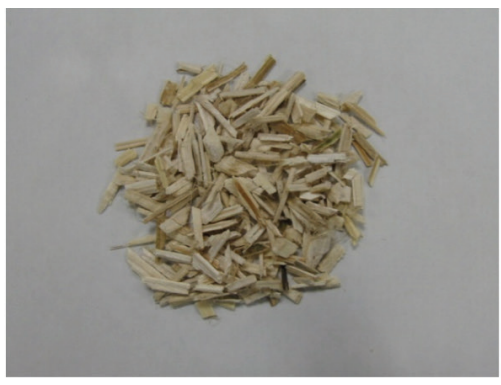

(b) Core

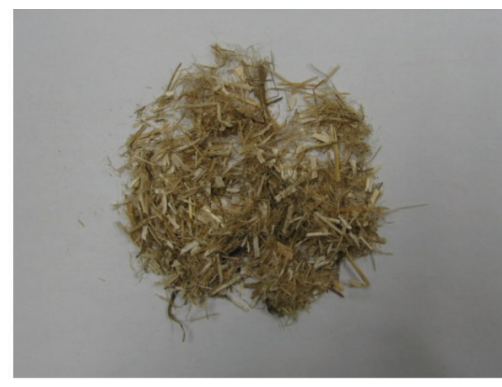

(c) Chaff

Fig. 3. Different fractions of the feedstock. 
Table 2. Microproperties of ball and bond used for simulations.

\begin{tabular}{lcc}
\hline \multicolumn{1}{c}{ Microproperty } & \multicolumn{2}{c}{ Value } \\
\cline { 2 - 3 } & Fibre & Core \\
\hline Ball & & \\
$\quad$ Normal stiffness $\left(K_{n}\right), \mathrm{N} \mathrm{m}^{-1}$ & $5.0 \times 10^{4}$ & $8.0 \times 10^{4}$ \\
$\quad$ Shear stiffness $\left(K_{s}\right), \mathrm{N} \mathrm{m}^{-1}$ & $5.0 \times 10^{4}$ & $8.0 \times 10^{4}$ \\
$\quad$ Friction $(\mu)$ & 1.0 & 1.0 \\
Bond & & \\
$\quad$ Normal stiffness $\left(\bar{k}^{n}\right), \mathrm{Pa} \mathrm{m}^{-1}$ & $1.0 \times 10^{14}$ & $2.5 \times 10^{9}$ \\
$\quad$ & $1.0 \times 10^{14}$ & $2.5 \times 10^{9}$ \\
$\quad$ Shear stiffness $\left(\bar{k}^{s}\right), \mathrm{Pa} \mathrm{m}^{-1}$ & & \\
$\quad$ Normal strength $\left(\sigma_{c}\right), \mathrm{Pa}$ & $3.6 \times 10^{8}$ & $2.2 \times 10^{6}$ \\
$\quad$ Shear strength $\left(\tau_{c}\right), \mathrm{Pa}$ & $3.6 \times 10^{8}$ & $2.2 \times 10^{6}$ \\
\hline
\end{tabular}

arranged in series, and the balls are connected with cylindrical bonds (Fig. 4a). The virtual core was approximated by a cluster of two layers of balls (Fig. 4b), with one layer having slightly larger dimensions than the other to simulate the curved cross-section of core. Bonds also connect balls in a virtual core. The ball and bond model were implemented in $\mathrm{PFC}^{\mathrm{BD}}$, named parallel bond model. Bonds between balls provide some strength to the structure of the material to be simulated.

All balls in a virtual fibre cluster have the same diameter, which was set as the diameter of the fibre to be simulated. The number of balls in a virtual fibre can be determined based on the fibre length using the following equation

$$
n_{f}=\frac{l}{D_{f}}+1
$$

where:

$n_{f}=$ number of balls in a virtual fibre;

$D_{f}=$ diameter of ball a virtual fibre $(\mathrm{mm})$;

$l=$ length of virtual fibre $(\mathrm{mm})$.

The ball diameter of the core was set to be half of the core thickness. The total number of balls required for a virtual core depends on the width and length of the core as follows:

$$
n_{c}=\frac{b_{c 1} l_{c 1}+b_{c 2} l_{c 2}}{D_{c}^{2}}
$$

where:

$n_{c}=$ number of particles in a virtual core;

$D_{c}=$ diameter of ball in a virtual core $(\mathrm{mm})$;

$b_{c 1}, b_{c 2}=$ width of the bottom and top layer of virtual core $(\mathrm{mm})$;

$l_{c 1}, l_{c 2}=$ length the bottom and top layer of virtual core (mm).

Model microproperty The virtual fibre and core described above are defined by a set of ball and bond microproperties. In $\mathrm{PFC}^{3 \mathrm{D}}$, the ball microproperties include normal stiffness $\left(K_{n}\right)$, shear stiffness $\left(K_{s}\right)$, and friction coefficient $(\mu)$, and the bond microproperties include normal and shear stiffness $\left(\bar{k}^{n}\right.$ and $\left.\bar{k}^{s}\right)$, normal and shear strength $\left(\sigma_{c}\right.$ and $\left.\tau_{c}\right)$, and the radius of the (a)
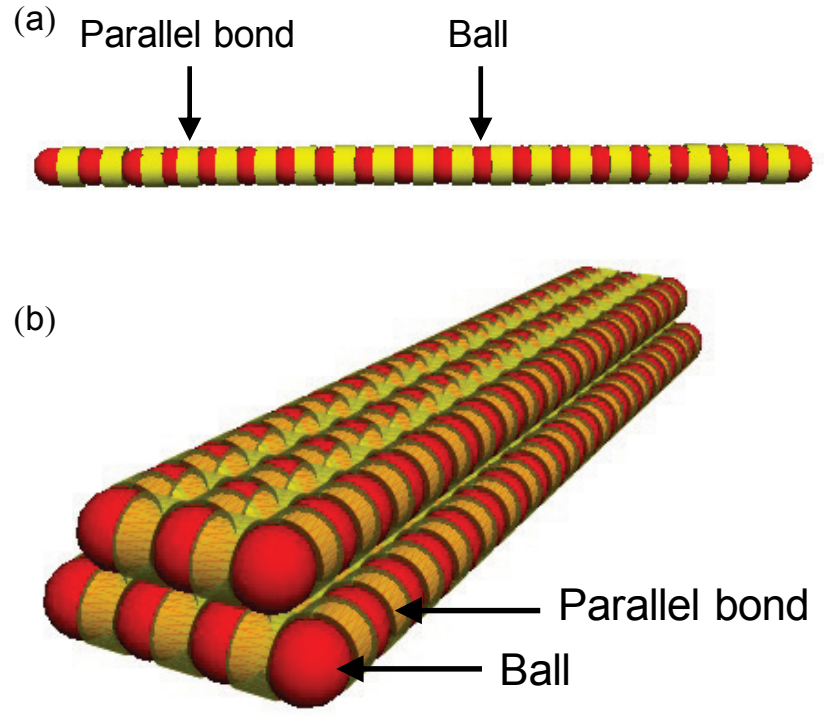

Fig. 4. Virtual hemp material: (a) virtual fibre, (b) virtual core.

cylindrical bond $(R)$. The $\mathrm{R}$ was considered to be equal to the ball diameter, and the other microproperties have been previously calibrated for both hemp fibre and core (Sadek et al. 2011; Sadek 2013). Those calibrated values were used in this study and they are summarised in Table 2. Virtual 3D separator A virtual 3D separator (Fig. 5) was constructed using the $\mathrm{PFC}^{3 \mathrm{D}}$ wall logic. The main body of the separator was simplified as a cylindrical wall. The screen was formed using intersecting lines with an equal spacing of $20 \mathrm{~mm}$. The screen was placed at the two-third height of the main body from the bottom. A flat wall was placed at the bottom of the main body to function as the collecting pan of the separator.

Generation of virtual hemp sample To simulate the separation process, a mixture of fibres, cores, and chaff needed to be generated in the virtual separator. In $\mathrm{PFC}^{3 \mathrm{D}}$, it is difficult to directly generate clusters, which were used to define the virtual fibres and cores in this study. Therefore, several steps were followed. The first step was to generate the desired number of individual balls on the top of the screen at the centre of the virtual separator. The second step was to replace each of those balls by a virtual fibre or core. In the first step, the generated balls were assigned to a group name: "fibre" and "core". Thus, each group could be replaced accordingly by a group of virtual fibres or a group of virtual cores. The ratio of the two groups could be adjusted for the desired compositions of fibre and core in the mixture. Within a group, the particle size can be varied to reflect the real particle size distribution. Figure 6a shows a mixture generated at the centre of the virtual separator before the separation process starts. Enlarged top view of the mixture (Fig. 6b) illustrates that the fibre and core clusters are well mixed in the mixture and their orientations are random in nature, representing a real situation. A virtual fibre or a core shorter than $20 \mathrm{~mm}$ is considered as chaff. Chaff also includes individual balls ranging from 0.30 to $2.0 \mathrm{~mm}$ in diameter. 


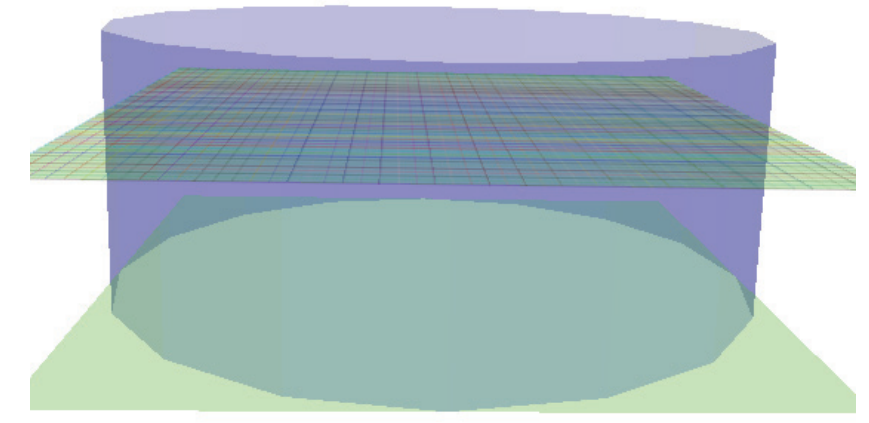

Fig. 5. Virtual 3D vibratory separator.

Motion of the virtual separator The horizontal and vertical motion of the virtual separator was set the same as the real 3D vibratory separator. The velocities were calculated using the angular frequency (w) and vibration amplitude (A) of the separator. The angular frequency was $40 \pi$, derived from the rational speed of the motor (1200 rpm), and the amplitudes varied with the separator settings, and they were obtained from the tests. The vibrating function is expressed by

$$
\begin{aligned}
& \mathrm{V}_{\mathrm{x}}=\mathrm{A} \cos (\omega \mathrm{t}) \mathrm{V}_{\mathrm{z}}=\mathrm{A} \sin (\omega \mathrm{t}+\phi) \\
& \mathrm{V}_{\mathrm{y}}=\mathrm{A} \cos (\omega \mathrm{t}) \\
& \mathrm{V}_{\mathrm{z}}=\mathrm{A} \sin (\omega \mathrm{t}+\phi)
\end{aligned}
$$

where:

$\mathrm{v}_{\mathrm{x}}, \quad \mathrm{v}_{\mathrm{y}}$ and $\mathrm{v}_{\mathrm{z}}=$ translational velocities of the virtual separator in $\mathrm{x}, \mathrm{y}$ and $\mathrm{z}$ directions, respectively $(\mathrm{m} / \mathrm{s})$;

$\mathrm{A}=$ vibration amplitude $(\mathrm{m})$;

$\omega=$ angular frequency (radian);

$\varphi=$ lead angle (radian);

$\mathrm{t}=$ time $(\mathrm{s})$.

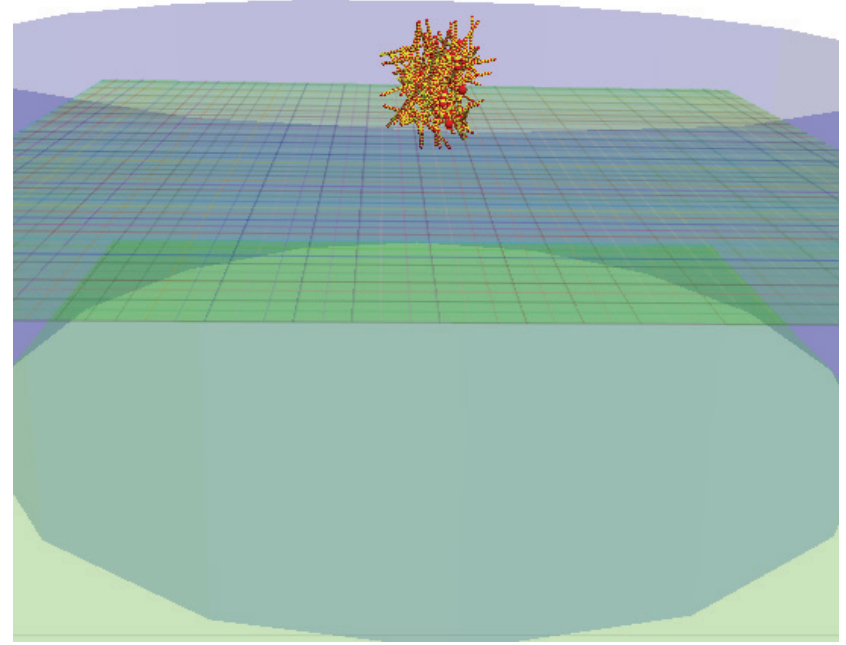

(a) Side view

Fig. 6. Virtual hemp sample on the screen.
Before the simulations of the separation process, the model was tested to examine if particles would follow the expected motion pattern. For this purpose, a single virtual fibre was generated at the centre of the screen (Fig. 7a). After the vibratory motion was introduced, a spiral motion of the fibre was observed. The stationary fibre started moving outwards (Fig. 7b), then touching the periphery (Fig. 7c), and finally traveling along the periphery (Fig. 7d). This motion pattern was similar to that described by the supplier of the real separator.

\section{RESULTS AND DISCUSSION}

Results from the separation tests

Composition of the feedstock Within the feedstock, the largest fraction was the fibre, which was accounted for $59 \%$ of total mass. There was only $15 \%$ of core. The remaining $26 \%$ was chaff.

Measured separation efficiency Separation efficiency was determined using Eq.1 and the efficiency data were statistically analyzed. The statistical analysis showed that the experimental factors, horizontal vibration force and its interaction with the vertical vibration force, significantly affected the separation efficiency (Table 3), whereas the vertical vibration force did not significantly affect the efficiency. Therefore, the simple effects of the experimental factors are presented due to the significance of their interactions.

The separation efficiency of the $3 \mathrm{D}$ vibratory separator was highly variable, from 23.4 to $86.2 \%$. (Fig. 8). Overall, the efficiency of the separator was lower. Some chaff might have been trapped in the tangled fibres, which prevented them from passing through the screen. As for the treatment effects, increasing the horizontal force for all levels of vertical vibration force increased the separation efficiency. There was no clear trend of the efficiency while changing the vertical vibration force. The highest separation efficiency, $86.2 \%$, was observed when using force setting H1V2, followed by the combination of

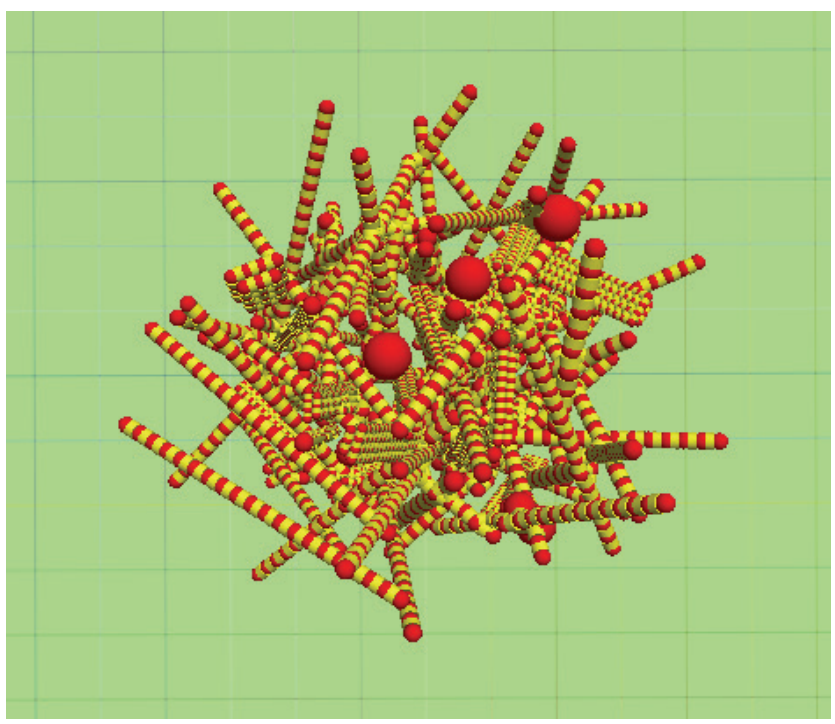

(b) Enlarged top view 


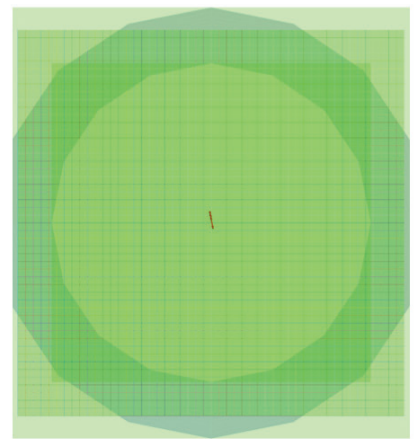

(a)

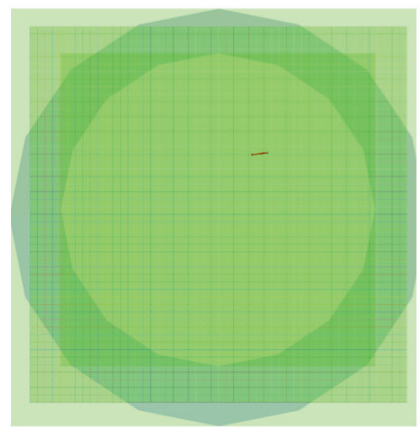

(b)

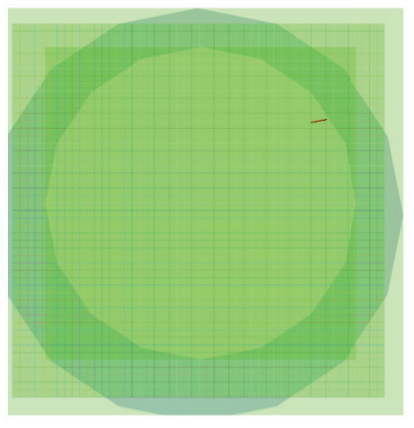

(c)

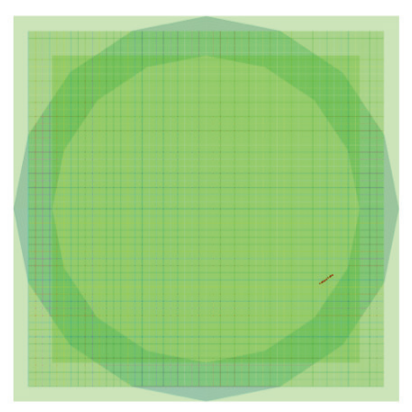

(d)

Fig. 7. Screenshots of the motion of a single particle at different simulation times.

H1V3. The lowest efficiency, $23.4 \%$, was obtained when using the V2H3 combination. For the H1V2 treatment, the majority particles $(83 \%)$ in the portion which passed the screen was chaff. it contained only $7 \%$ of fibre particles, and the rest $10 \%$ was core particles, which were larger than the screen opening size. Those $17 \%$ larger particles might not have been completely in horizontal positions on the screen, and therefore they fell through the screen. This phenomenon would be expected in an application of granular material separation using a $3 \mathrm{D}$ vibratory separator. Also, the passing of the non-chaff fraction in this study would be highly variable, as hemp particles undergoing vibration had random orientations. Further study on particle orientation on the screen could be useful in understanding the separation efficiency of the separator.

\section{Results from simulations}

Conditions of simulation The model was run to simulate the separation process of decorticated hemp using the 3D vibratory separator. The virtual separator was operated to simulate the best setting (H1V2) observed in the tests. The vibration magnitude of the virtual separator was set as 3.9 $\mathrm{mm}$ for both horizontal and vertical directions, measured in the tests for the setting. Equations 4, 5, and 6 determined the horizontal and vertical motions of the virtual separator. Its cyclic motion in the model is demonstrated in Fig. 9. The phase difference between the horizontal and vertical directions was caused by the lead angle recommended by the manufacturer of the separator.

For simplicity, all virtual fibres had a constant diameter of $0.30 \mathrm{~mm}$ (Sadek 2013), and all virtual cores had a thickness and a width of $4 \mathrm{~mm}$ and $10 \mathrm{~mm}$ respectively (Khan et al. 2010). The lengths of the virtual fibres and cores were made variable. In total, six different

Table 3. ANOVA results on the effects of experimental factors.

\begin{tabular}{lcrrr}
\hline Source & df & MS & F & \multicolumn{1}{c}{ P } \\
\hline Horizontal & 2 & 6304.66 & 67.79 & $<0.0001$ \\
Vertical & 2 & 92.52 & 0.99 & 0.3829 \\
Horizontal*Vertical & 4 & 590.67 & 6.35 & 0.001 \\
Error & 27 & 92.99 & & \\
\hline
\end{tabular}

particles (Table 3) were formed, and they were long fibre (Fibre A) and long core (Core A), short fibre (Fibre B) and short core (Core $\mathrm{B})$, which were all greater than the screen opening size $(20 \mathrm{~mm})$ of the separator. The other two particles were chaff (Fibre Chaff and Core Chaff) or individual balls, which were all smaller than $20 \mathrm{~mm}$ in size, so that they were capable of passing the screen. The dimensions of the different compositions are listed in Table 4. These particles were used to make different composite samples of virtual hemp particles for the separation simulations. All simulation lasted for three minutes as in the tests. During the course of simulation, the masses of passing particles were recorded using the $\mathrm{PFC}^{3 \mathrm{D}}$ fish function to investigate the separation rate and efficiency.

Model validation For model validation, a virtual sample of processed hemp was created using 59\% of fibre (including $48 \%$ of Fibre A and $11 \%$ of Fibre B), $15 \%$ of Core (including $4 \%$ of Core A and $11 \%$ of Core B), and $26 \%$ of chaff (including 13\% of Fibre Chaff and 13\% of

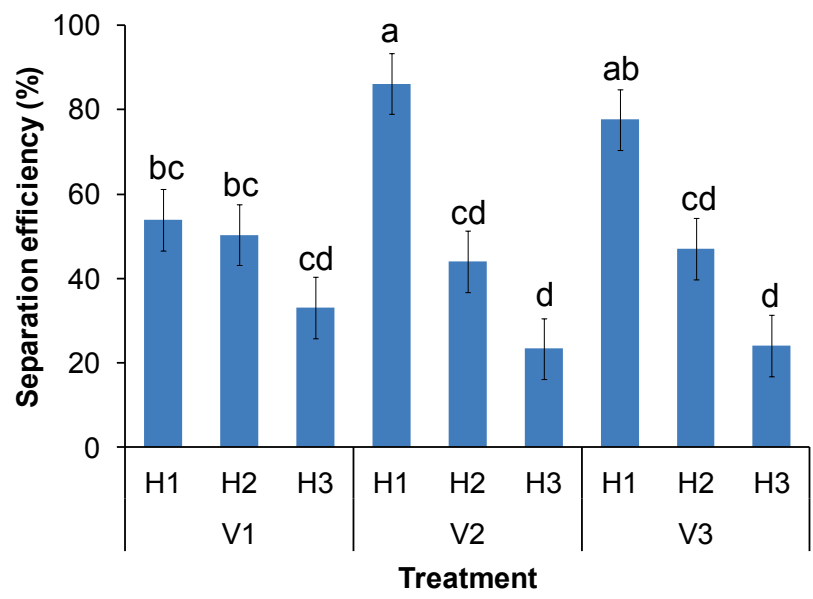

Fig. 8. Separation efficiencies of the 3D vibratory separator for different vibration forces. $\mathrm{H} 1, \mathrm{H} 2$, $\mathrm{H3}$ = levels of horizontal force, V1, V2, V3 = levels of vertical force. Means followed with different letters are significantly different; error bars stand for standard errors. 


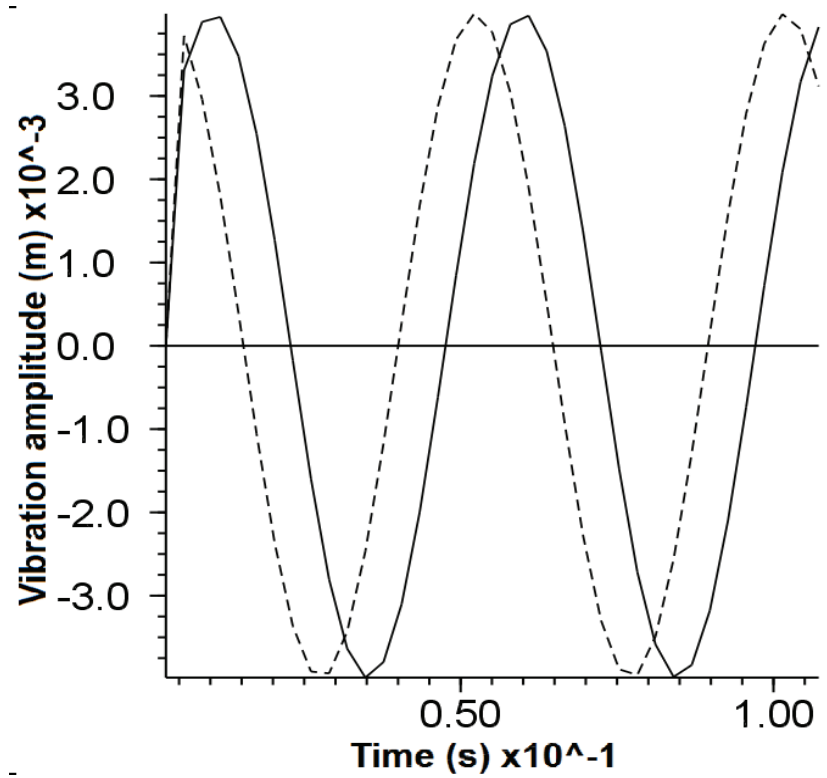

Fig. 9. Vibratory motion of the virtual separator; the left curve is the motion in the $x$ and $y$ directions and the right is the motion in the $z$ direction.

Core Chaff), so that this virtual sample had the same composition as that used in the test conducted for model validation. The virtual sample also had a total weight of $100 \mathrm{~g}$ as the sample in the test. All these ensured that simulation results could be compared with the test results.

As the virtual separator vibrated, small virtual particles quickly fell through the screen. Most particles on the screen had sliding motion, while few particles were "jumping" on the screen. Some of the jumping particles may orientate vertically, which resulted in them passing through the screen, even though their lengths were greater than the screen opening size, as illustrated in Fig. 10. This phenomenon reflects separation processes in reality.

The percentage of passing particles monitored during the simulation was plotted in Fig. 11, together with the measurement. Once the vibratory separator was started running, the percentage of passing particles increased linearly between 0 and 0.5 minute for both the simulation and measurement (Fig. 11). Those particles were likely the loose small particles in the sample. After that, the separation rate was slowed down, and the curve remained gradually increasing to the end of 3 minutes. From 1 to 3

Table 4. Different particles used for forming virtual samples of processed hemp.

\begin{tabular}{ll}
\hline Particle & Dimension, mm \\
\hline Fibre A & $0.30 \times 60$ (Diameter x Length) \\
Fibre B & $0.30 \times 30$ (Diameter x Length) \\
Fibre Chaff & $\begin{array}{l}0.30 \times 10 \text { (Diameter x Length) and balls (0.30- } \\
\text { Core A }\end{array}$ \\
$\begin{array}{l}2.0 \mathrm{~mm} \text { in diameter) } \\
\text { Core B }\end{array}$ & $4 \times 10 \times 50$ (Thickness x Width x Length) \\
Core Chaff & $4 \times 10 \times 10$ (Thickness x Width x Length) \\
& balls $(0.30-2.0$ mm diameter)
\end{tabular}

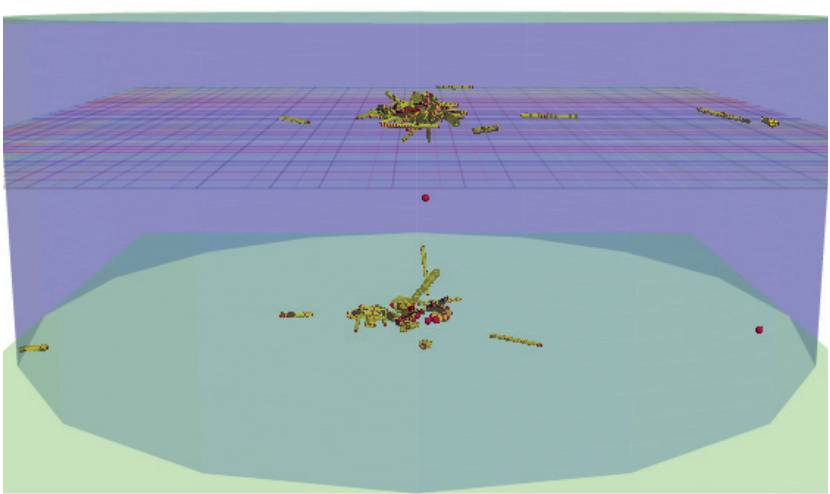

Fig. 10. A snapshot of virtual separation showing particles falling through the screen onto the collection pan at the bottom.

minutes, most passing particles were likely those which were initially trapped in the long fibres and cores, and being freed by the vibration motion, and falling through the screen gradually as the separator vibrated.

During the period from the beginning to 1 minute, the simulation and measurement showed similar separation rate. After 1 minute, the simulation had a higher separation rate. At the end of separation, the passing particles were accounted for $22 \%$ of the total mass of the sample in the test and $33 \%$ in the virtual separation. Given the 26\% chaff in the hemp sample, the results indicated that not all chaff particles have passed through the screen in the test. This maybe attributable to the entangled fibres in which chaff particles were held. The results also indicated that particles other than chaff have passed through the screen in the virtual separation. The explanation for the higher percentage of passing particles in the virtual separation was that the virtual particles would have little or no tangling problem. As the result, particles greater than the screen size passed through the screen, when they oriented vertically.

Model applications Simulations of separation rate were conducted for other two different virtual samples. Sample 1 was composed $69 \%$ of fibre, $15 \%$ of core, and $16 \%$ of chaff, and Sample 2 was composed $49 \%$ of fibre, $15 \%$ of core, and $36 \%$ of chaff, as listed in Table 5. Sample 1 had less chaff and more long fibres than Sample 2. These two Samples were arbitrarily formed to investigate whether the separation rates of two contrasting samples were different.

Table 5. Composition of the virtual samples of processed hemp.

\begin{tabular}{lcc}
\hline & \multicolumn{2}{c}{ Percentage } \\
\cline { 2 - 3 } Particle & Sample 1 & Sample 2 \\
\hline Fibre A & 58 & 38 \\
Fibre B & 11 & 11 \\
Fibre Chaff & 8 & 18 \\
Core A & 4 & 4 \\
Core B & 11 & 11 \\
Core Chaff & 8 & 18 \\
\hline
\end{tabular}




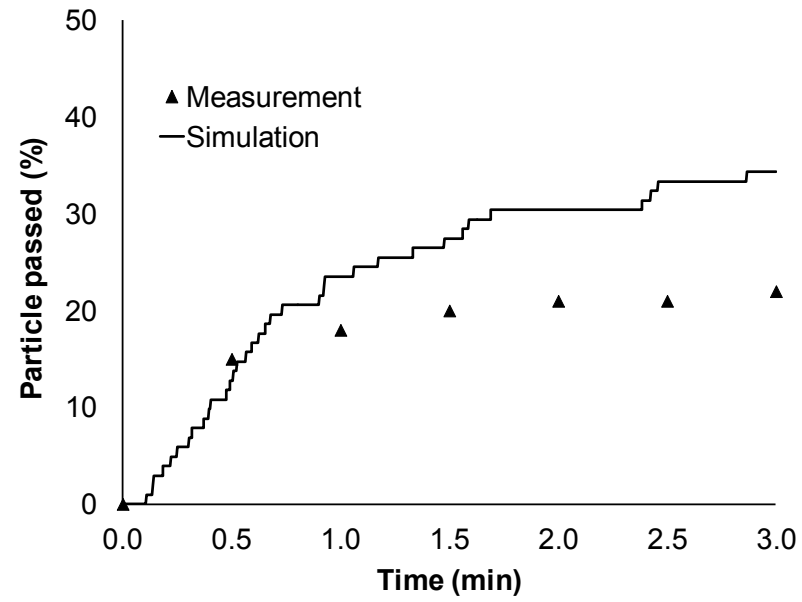

Fig. 11. Comparisons of separation rate between simulation and measurement; each value of the measurement curve is the average of three data points.

For Sample 1, the simulation results showed that the amount of passing particles was continuously increasing over time in a non-linear fashion during the entire separation period (Fig. 12). At approximately 1 minute, $16 \%$ particles have passed through the screen, meaning a $100 \%$ separation efficiency has been achieved at this point, given the chaff percentage in the sample of $16 \%$. At the end of 3 minutes, a total of $27 \%$ particles have passed through the screen. This implied that the passing particles included some non-chaff particles as well. Therefore, the separation duration for this sample should be no longer than 1 minute.

The separation rate of Sample 2 was different with that of Sample 1, due to the different compositions of two samples. During the separation of Sample 2, the percentage of passing particles was gradually increased over the period of the first minute; approximately $32 \%$ of the particles have passed the screen at that point, giving a separation efficiency of approximately $90 \%$. Then, the separation rate slightly increased over the rest of the separation duration. The $100 \%$ separation efficiency (i.e. $36 \%$ of passing particles) was achieved at approximately 2 minutes, given the $36 \%$ of chaff in the virtual sample. During the last minute of separation, only additional $4 \%$ of the particles passed through the screen. This implied that up to $4 \%$ of non-chaff particles could end up into the chaff portion.

In summary, the model results showed that the separation rate of the $3 \mathrm{D}$ separator was affected by the composition of the sample. Higher percentage of the small particles in a sample resulted in a more rapid separation rate. The model results can help determine the separation duration. To achieve $100 \%$ separation efficiency, separation duration for Sample 1 should be 1 minute. Longer separation will not only increase the use of energy, but also increase the loss of non-chaff particles. For Sample 2, the duration has to be doubled to achieve $100 \%$ efficiency; however, one may choose 1-minute duration, if

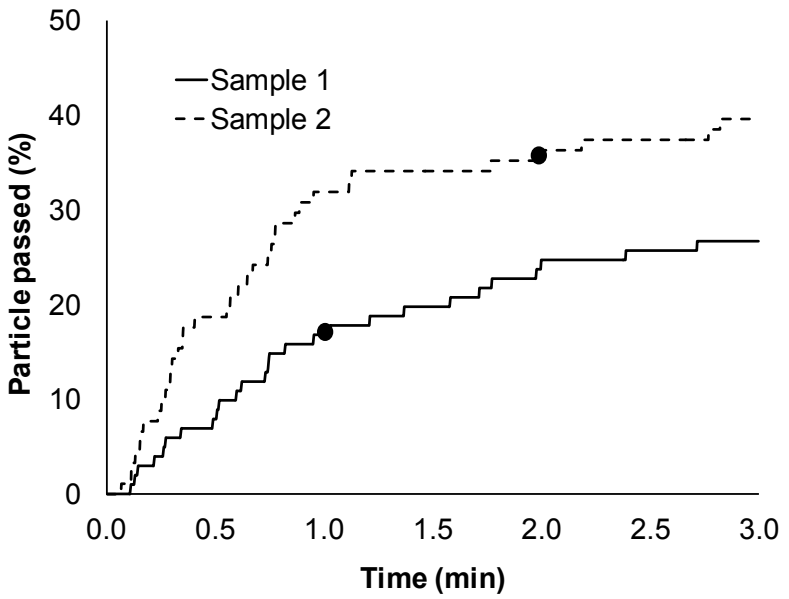

Fig. 12. Separation rates for two samples with different particle compositions; the dots stand for the $100 \%$ separation efficiency.

the $90 \%$ separation efficiency is considered to be sufficient. Significantly amount of energy could be saved with scarifying only $10 \%$ of the separation efficiency.

The model does not address the nature of the fibre entanglement. Therefore, it is expected that the model will give higher separation rates than measurements, under the same conditions. Caution should be taken when using the model. Also, the motion and orientation of hemp particles on a screen vibrating in three directions are a complicated regime. Detail examinations of dynamic behaviours of hemp particles on the vibrator screen will be carried out in future studies.

\section{CONCLUSION}

Tests of the $3 \mathrm{D}$ vibratory separator were conducted to remove the chaff from the processed hemp material. The results showed that the separation efficiency of the separator varied with different vibration settings. The highest separation efficiency was found to be $86.2 \%$. In simulations of the separation process of the $3 \mathrm{D}$ vibratory separator, hemp fibre and core can be defined as $\mathrm{PFC}^{3 \mathrm{D}}$ clusters. Different compositions of virtual processed hemp can be formed with those clusters having different sizes. Varying the composition of the virtual sample altered the simulated separation rate with the virtual sample containing more chaff particles having higher separation rate. Regardless of the composition of the virtual sample, sufficient separation efficiency could be achieved at approximately 1 minute after separation starts. Longer separation duration will have slower separation rate and cause some losses of non-chaff particles as well. The model developed in this study can simulate the general behaviour of hemp fibre, core, and chaff particles in the separation process using the $3 \mathrm{D}$ vibratory separator. However, the model did not address the fibre entanglement nature. As the result, the model over predicted the separation rate of processed hemp material, when compared to the measurements. 


\section{ACKNOWLEDGEMENT}

This research was financially supported by Natural Sciences and Engineering Research Council of Canada and Composites Innovation Centre, Manitoba, Canada.

\section{REFERENCES}

Anthony, W.S. 2002. Separation of fiber from seed flax straw. Applied Engineering in Agriculture 18(2): 227-233. http://dx.doi.org/10.13031/2013.7788

Anthony, W.S. 2005. Development of machines to separate fiber and shive from seed flax straw. Applied Engineering in Agriculture 21(6): 1057-1063. http://dx.doi.org/10.13031/2013.20022

Cleary, P. W., M. D. Sinnott and R. D. Morrison. 2009. Separation performance of double deck banana screens - Part 1: Flow and separation for different accelerations. Minerals Engineering 22:1218-1229. http://dx.doi.org/10.1016/j.mineng.2009.07.002

Cundall, P.A. and O.D.L. Strack. 1979. A discrete numerical model for granular assemblies. Geotechnique 29(1): 47-65. http://dx.doi.org/10.1680/geot.1979.29.1.47

Fürll, C. and H. Hempel. 2000. Optimisation of a new machine for fibre processing by impact stress. Research report, Potsdam-Bornim, Germany: Instiyut fur Agrartechnik.

Khan M.R., Y. Chen, C. Lague, H. Landry, Q. Peng and W. Zhong. 2010. Compressive properties of Hemp (Cannabis sativa L.) stalks. Biosystems Engineering 106(3): 315 - 323. http://dx.doi.org/0.1016/j.biosystemseng.2010.04.004

Münder, F., Ch. Fürll and H. Hempel. 2004. Advanced Decortication Technology for Unretted Bast Fibres. Journal of Natural Fibers 1(1): 49 - 65. http://dx.doi.org/10.1300/J395v01n01_04

Murariu, V. and P.J. Sergeant. 2007. Modeling of the separation process in a ferrohydrostatic separator using discrete element method. Physical Separation in Science and Engineering 2007: 1-13. http://dx.doi.org/10.1155/2007/48238
Parvin, S., Y. Chen, C. Laguë, H. Landry, Q. Peng and W. Zhong. 2013. Post-decortication cleaning of hemp fibre using selected methods. AES Technical Reviews International Journal, Part C: International Journal of Advances and Trends in Engineering Materials and their Applications 1(1): 53-65.

Pecenka R., C. Furll. 2008. Simulation of natural fibre cleaning in a comb shaker by means of a geometricstatistical model. ISHS Acta Horticulturathe e 802: 205-211.

Richardson, J.F., J.H. Harker and J.R. Backhurst. 2002. Coulson and Richardson's Chemical Engineering, Particle Technology \& Separation Processes, 5th edition. Tutterworth Heinemann, New York.

Sadek, M. A. 2013. Modeling biofibre (hemp) processing using the discrete element method (DEM). Unpublished Doctoral dissertation. Winnipeg, MB: Department of Biosystems Engineering, University of Manitoba.

Sadek, M. A., Y. Chen, C. Laguë, H. Landry, Q. Peng and W. Zhong. 2011. Characterization of the shear properties of hemp using discrete element method. Transaction of ASABE 54(6): 2279-2285. http://dx.doi.org/10.13031/2013.40641

Sakaguchi, F., M. Suzuki, J.F. Favier and S. Kawakami. 2001. Numerical simulation of the shaking separation of paddy and brown rice using the discrete element method. Journal of Agricultural Engineering Research 79(3): 307-315. http://dx.doi.org/10.1006/jaer.2001.0706

Sweco. 2003. XS40 Vibro Energy Separator, Installation, Operation and Maintenance Manual. SWECO Canada, Inc., Markham, ON, Canada.

Vorster, W., A. Hinde and F. Schiefer. 2002. Increased screening efficiency using a kroosher unit coupled with a sweco screen (Part 1). Minerals Engineering 15(1-2): 107-110. http://dx.doi.org/10.1016/S0892-6875(01)00210-2 